\title{
Proposta de um modelo conceitual para minimizar as dificuldades no uso do QFD
}

\author{
José Antonio Carnevalui \\ UNIMEP \\ Paulo Augusto Cauchick Miguel \\ EPUSP \\ Felipe Araújo Calarge \\ UNINOVE
}

\section{Resumo}

Este trabalho apresenta uma proposta de desenvolvimento de um modelo de aplicação do QFD, com o objetivo de diminuir as dificuldades do uso do método. Para desenvolver este modelo foram realizadas as seguintes atividades: análise da literatura buscando identificar as informações sobre o uso do QFD, análise dos resultados de um levantamento de campo e de revisão de dados de entrevistas em empresas que utilizaram o método e da aplicação do AD (Axiomatic Design) para desenvolver o modelo conceitual proposto com os dados das etapas anteriores. As principais dificuldades identificadas a serem resolvidas estão relacionadas à matriz da qualidade, ao trabalho em equipe, ao modelo conceitual (do próprio QFD) e à avaliação da aplicação do QFD. Os resultados indicam a viabilidade do uso do AD para atender a este objetivo, mas o trabalho conclui que uma validação empírica é necessária.

Palavras-chave

Desdobramento da Função Qualidade (QFD) Projeto Axiomático.

\section{Proposal of a conceptual model to minimize QFD application difficulties}

\begin{abstract}
This work presents a proposal of a conceptual model of QFD application aiming at minimizing the difficulties in the use of the method. To develop this model, the following stages were carried out: literature review to identify information about the QFD use, analysis of a field research and review of previous interview data from QFD users, and the use of $A D$ (Axiomatic Design) to develop the suggested model with the data of previous stages. The main difficulties which were identified and yet to be solved are related to quality matrix, team work, QFD conceptual model and QFD application assessment. The results indicate the feasibility of the $A D$ use to achieve this objective but the work concludes that an empirical application is needed for validity.
\end{abstract}

Key words

Quality Function Deployment (QFD); Axiomatic Design. 


\section{INTRODUĈ̣̃O}

Num ambiente de mercado cada vez mais globalizado, onde o cliente tem uma grande oferta de produtos e serviços, torna-se necessário que as empresas desenvolvam produtos que ganhem a preferência do cliente. O Desdobramento da Função Qualidade (QFD) é um importante método que atende este objetivo, melhorando a confiabilidade do produto, reduzindo o tempo de projeto (DEVADASAN et al., 2006) e aumentando a satisfação dos clientes (LAGER, 2005).

Entretanto, as empresas enfrentam várias dificuldades na introdução e utilização do método, tais como: dificuldade de interpretar a "voz do cliente" (CHAN; WU, 2005), priorizar as características da qualidade (KARSAK, 2004), definir a qualidade projetada (RAMASAMY e SELLADURAI, 2004), trabalhar com matrizes grandes (DIKMEN et al., 2005) e, finalmente, dificuldades geradas pelo trabalho em equipes (LOWE e RIDGWAY, 2000), dentre outras.

Tais dificuldades têm desmotivado a aplicação do QFD e diminuído sua eficácia. Assim, torna-se importante desenvolver estudos que busquem eliminar ou pelo menos diminuir estas dificuldades. O objetivo fundamental deste trabalho é desenvolver um modelo conceitual de aplicação do QFD visando reduzir/eliminar algumas das dificuldades que as empresas enfrentam ao usar o QFD. O termo modelo conceitual é considerado no presente trabalho como um "framework" que representa as dificuldades e soluções para estas no uso do QFD, onde o modelo representa dois domínios: o do problema (dificuldades) e o da solução, estruturado pelo Projeto Axiomático (Axiomatic Design). Este modelo deve também orientar as etapas fundamentais na aplicação do método. O presente trabalho pode ser caracterizado como teórico-conceitual pois, segundo a indicação de Oliveira (1997), a pesquisa teórica tem como objetivo desenvolver modelos e sistemas teóricos, como no caso deste estudo, que utiliza múltiplos métodos. Um modelo é a representação do sistema em estudo podendo ser usado para aplicações teóricas ou empíricas (COOPER; SCHINDLER, 2003). Para a construção deste modelo é apresentada a seguir a abordagem metodológica do presente trabalho.

\section{ABORDAGEM METODOLÓGICA}

Primeiramente, cabe destacar que a abordagem metodológica do presente trabalho é de multimétodo. Assim, para desenvolver o modelo conceitual proposto é necessário realizar o conjunto de atividades apresentado na Figura 1.
A estrutura apresentada na Figura 1 compreende as seguintes atividades:

Atividade 1: Análise da literatura sobre o QFD usando o diagrama de afinidades com o diagrama em árvore, para organizar os dados sobre benefícios, pré-requisitos, dificuldades e recomendações de aplicação do QFD;

Atividade 2: Identificação das expectativas, dificuldades e atributos no uso do QFD, realizando uma revisão de dados oriundos de entrevistas em empresas que usam o método. Estas entrevistas fizeram parte de uma pesquisa anterior, realizada no passado, que tinha um outro escopo. Por esta razão, foi realizada uma nova pesquisa de campo, para coletar dados específicos para aplicação do AD. Esta nova pesquisa foi realizada com empresas usuárias do QFD;

\section{mbora o QFD seja um método eficaz para dar suporte ao desenvolvimento de novos , é importante desenvolver estudos que busquem eliminar ou pelo menos diminuir as dificuldades na sua aplicação.}

Atividade 3: Aplicação da abordagem do Projeto Axiomático para desenvolver o modelo proposto com os dados coletados e tratados nas atividades anteriores 1 e 2 ;

Atividade 4: Utilização do diagrama de módulo e junção para definir a seqüência de aplicação do modelo.

No próximo tópico são apresentados os resultados de cada uma destas atividades.

\section{Revisão de Literatura sobre o QFD}

Seguindo as etapas sugeridas por Marconi e Lakatos (2002) realizou-se uma pesquisa bibliográfica sobre o QFD por meio dos seguintes passos:

1. Identificação e localização: consulta via bibliotecas, CDROM de congressos; sites de bibliotecas (UNICAMP e USP), portal dos periódicos da CAPES e livros sobre a teoria do QFD;

2. Obtenção: os textos obtidos via arquivos eletrônicos do portal de periódicos da CAPES; impressão de artigos de congressos em CD-ROM e empréstimos das bibliotecas, obtidos via COMUT (Programa de Comutação Bibliográfica);

3. Cadastramento das fontes bibliográficas: fichamento dos textos de interesse utilizando-se de uma adaptação do formulário de Martín et al. (1999). Foram cadastrados os artigos de periódicos publicados entre 2000 a 2006 considerando um horizonte de análise de sete anos, pois existiam textos relevantes com mais de cinco anos. Os livros consultados não foram fichados, mas apenas estudados para 
dar uma base teórica para o desenvolvimento da proposta. Os artigos de congressos e simpósios não foram cadastrados, priorizando-se os textos de periódicos por serem publicados após passarem por uma avaliação criteriosa. No total foram cadastrados 167 artigos em periódicos.

Em relação aos dados sobre o QFD identificados nos artigos, estes foram analisados utilizando-se o diagrama de afinidades e o diagrama em árvore de modo a organizar, agrupar e separar em tabelas individuais as informações sobre: os pré-requisitos para aplicar o método, as dificuldades do uso do método, quais benefícios o seu uso gera e o que a literatura recomenda para eliminar ou diminuir as dificuldades identificadas sobre o uso do QFD.

\section{Desenvolvimento de um levantamento de campo [atual]}

Buscando completar os dados sobre o uso do QFD identificados na literatura, foi realizado um levantamento visando uma pesquisa de campo para identificar as dificuldades e benefícios da utilização do QFD e também os atributos e expectativas dos clientes do QFD, de modo a ajudar a definir o domínio do cliente, primeira etapa de aplicação do AD, descrita mais à frente.

Como esta coleta foi realizada em empresas, foram considerados como "clientes" do QFD os membros da equipe que o aplicam, os gerentes funcionais envolvidos com o projeto, mas que não estão na equipe e a alta administração da empresa. A amostra do estudo foi composta por 21 empresas que participaram de um levantamento realizado no Brasil (MIGUEL, 2003) e que utilizaram o QFD, provenientes dos seguintes setores: automobilístico (3 empresas em SP), metalúrgica básica (3 empresas de diferentes estados: BA, MG e SP), eletrodomésticos (3 empresas de diferentes estados: RS, SC e SP), produtos químicos (duas empresas: na BA e em SP), máquinas e equipamentos (duas empresas: em SC e em SP), produtos alimentícios (duas empresas: em SC e em SP), produtos têxteis (uma empresa em SP), serviços: vendas, manutenção e modernização de elevadores (uma empresa em SP), produtos e serviços

Figura 1: Estrutura das atividades realizadas.

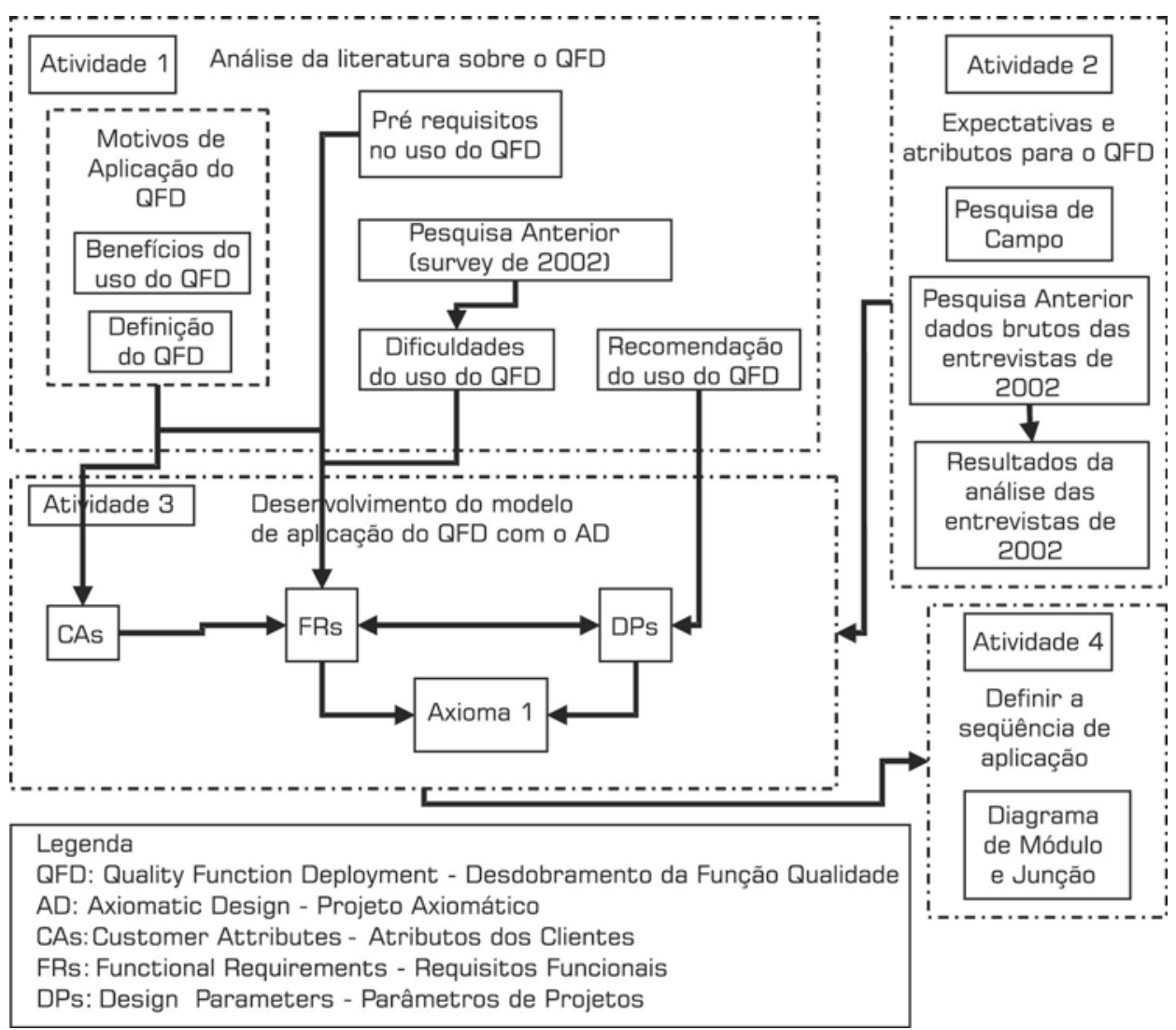


ferroviários (uma empresa em MG), materiais de construção (uma empresa em SC), embalagens (uma empresa em $\mathrm{SP}$ ), produtos elétricos (uma empresa em SP). Utilizou-se a técnica de coleta de dados via questionários, enviados entre o final de 2005 e início de 2006. Para a análise dos dados, foi necessário codificar somente as questões abertas do questionário, sendo todos os resultados tabulados em planilha eletrônica do Excel $^{\circledR}$.

\section{Revisão dos dados de um levantamento de campo realizado anteriormente pelos autores}

Buscando aprofundar um levantamento anterior, foram realizadas quatro entrevistas em empresas de referência no uso do QFD, identificadas no levantamento tipo survey (CARNEVALLI et al., 2004), cujos resultados das entrevistas estão em Carnevalli e Miguel (2003). Foram entrevistados os mesmos indivíduos que participaram da pesquisa tipo survey e, em alguns casos, também outros membros da equipe que aplicava o QFD. As empresas entrevistadas estão localizadas no estado de São Paulo e são dos seguintes setores: alimentos; embalagens; automotivo; máquinas e equipamentos.

Para o presente trabalho, uma nova análise mais detalhada dos dados brutos contidos nas transcrições destas entrevistas foi realizada, buscando identificar outras expectativas do uso do QFD relacionadas a atributos dos clientes, de modo a ajudar na definição do domínio do cliente na aplicação do $\mathrm{AD}$, descrito a seguir.

\section{Projeto Axiomático}

O Projeto Axiomático (AD) é uma abordagem de desenvolvimento de projeto que busca gerar a melhor solução para um problema proposto (SUH, 1990). A abordagem envolve a definição de quatro domínios: domínio do cliente, que são os requisitos dos clientes para o projeto (CAs - Customer Attributes); domínio funcional, são os requisitos funcionais (FRs - Functional Requirements), que satisfazem os CAs; domínio físico, são os parâmetros de projetos (DPs - Design Parameters) que satisfazem os FRs; e o domínio do processo são as variáveis de processo (PVs - Process Variables) que atendem os DPs, conforme ilustrado na Figura 2. No presente trabalho não foi realizado o domínio do processo, pois o modelo proposto é o resultado do desenvolvimento do domínio funcional com o domínio físico.

Segundo Suh (1990), os FRs e DPs iniciam-se de um único item que vai sendo desdobrado em vários subitens mais detalhados, de forma hierarquizada. Neste processo, o projetista é orientado seguindo dois axiomas: o axioma da independência (axioma 1) e o da informação (axioma 2). No axioma $1 \mathrm{em}$ um projeto ideal, cada DP atende apenas a um FR independentemente da ordem de implementação (projeto uncoupled). Em um projeto aceitável, os DPs podem ser implementados em ordem pré-definida de modo que cada DP mantenha a independência de cada FR (projeto decoupled).

O axioma 1 pode ser representado por (SUH, 1990):

$\{\mathrm{FR}\}=[\mathrm{A}] \times\{\mathrm{DP}\}$

Onde: $\{F R\}$ é o vetor do FR; $\{\mathrm{DP}\}$ é o vetor do DP; e $[\mathrm{A}]$ é a matriz de projeto, que apresenta a relação existente entre FRs e DPs através dos seus elementos Aij, a qual pode ser projeto uncoupled (matriz diagonal) ou projeto decoupled (matriz triangular) para satisfazer o axioma 1, mas não projeto coupled conforme exemplificado na Tabela 1 onde, segundo Suh (1990), "x" indica a existência de dependência entre um FR com um DP.

Figura 2: Domínios do AD.

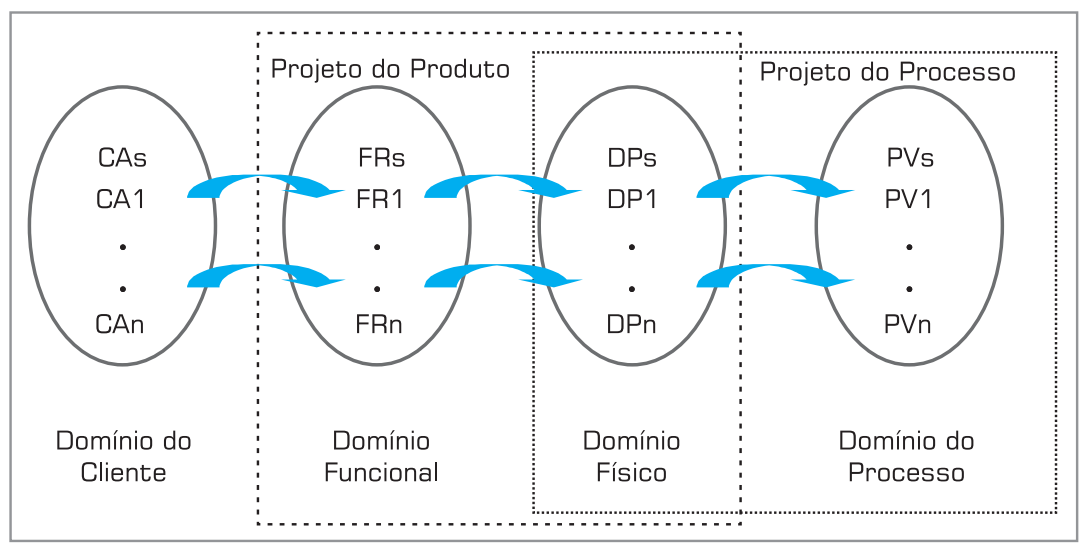


O axioma 2 é um modo de medir a quantidade de informação que um projeto necessita para ser realizado e o melhor projeto, em um conjunto de projetos, será aquele que atende o axioma 1 e tem necessidade de menos informações, ou seja é o projeto mais simples (SUH, 1990, 2001). No caso do presente trabalho não utilizou-se o axioma 2 já que foi desenvolvido apenas um único projeto.

Após a realização de todos os desdobramento das DPs e FRs, realiza-se a matriz de projeto consolidada (ou completa), que verifica o relacionamento entre "FRsfolha" (são os FR de mais baixo nível da estrutura hierarquizada) e o "DPs-folha". Com esta matriz consolidada, verifica-se o projeto como um todo atende as condições do axioma 1.

Para definir a seqüência de aplicação do modelo desenvolvido, foi utilizado o diagrama de módulo e junção que, de acordo com Suh (2001), mostra a ordem de implantação baseado no relacionamento da matriz de projeto e do detalhamento dos FRs e DPs.

A Figura 3 apresenta como este diagrama representa as relações internas entre os DPs e FRs na matriz de projeto. Nos casos em que os FRs são independentes (projeto uncoupled ou no caso do FR1 do projeto decoupled na Figura 3) o diagrama apresenta uma relação de soma (S) podendo se aplicar em paralelo os dois módulos; e caso que haja dependência entre os FRs (projeto decoupled), o diagrama mostra uma relação de controle $(\mathrm{C})$ sendo que o primeiro módulo deve ser aplicado antes do segundo.

O objetivo do uso do AD no presente trabalho é estabelecer a seqüência que as empresas devem seguir para aplicar o QFD, e recomendar melhores soluções existentes na literatura para eliminar ou reduzir as dificuldades de aplicação do método. O AD é, portanto, entendido como parte da abordagem metodológica adotada neste trabalho.

Tabela 1: Tipo de projeto.

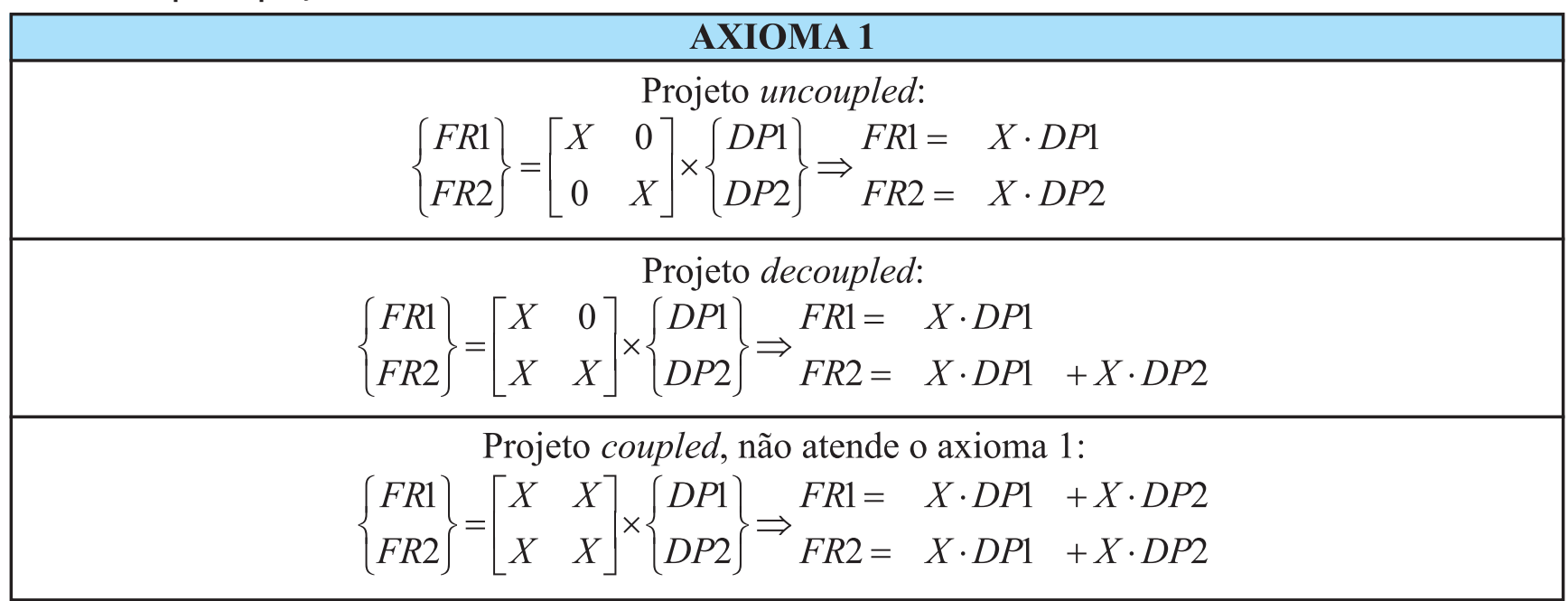

Fonte: adaptado de SUH, 1990

Figura 3: Exemplo do diagrama de módulo e junção da matriz 1.

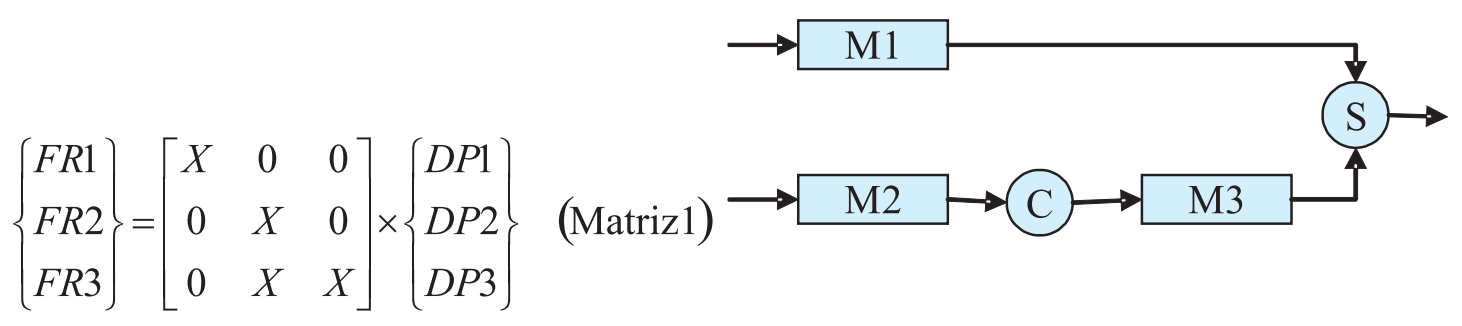

Fonte: adaptado de SUH, 2001 


\section{DESENVOLVIMENTO DO TRABALHO}

Neste tópico são descritos os resultados da realização das etapas do trabalho, cuja apresentação é estruturada conforme as atividades mostradas no item anterior.

\section{Análise dos dados de interesse sobre o uso do método do QFD}

Com a análise de 167 artigos foram identificadas 261 citações sobre os benefícios do uso do QFD, sendo que em $80 \%$ dos casos referem-se a benefícios intangíveis, tais como: "ajudar no trabalho em equipe" (GINN; ZAIRI, 2005) e "na melhoria da comunicação entre os departamentos" (RAMASAMY; SELLADURAI, 2004), dentre outros. Das 261 citações, apenas pouco mais de 19\% se referem a benefícios tangíveis do uso do QFD, o que pode indicar que os benefícios tangíveis ocorrem menos que os intangíveis. Tais benefícios referem-se a: "reduzir o número de falhas no projeto" (SHER, 2006), "redução dos custos de projeto" (LOWE; RIDGWAY, 2000), dentre outros.

$\mathrm{Na}$ análise da literatura foram identificadas 68 citações de pré-requisitos para aplicação do QFD, sendo que estes requisitos são similares aos encontrados em aplicações de métodos complexos. De maneira geral, estão relacionados à necessidade de apoio da alta administração (GINN; ZAIRI, 2005), de recursos (GUIMARÃES, 2003), de treinamento no método (DIKME et al., 2005), dentre outros. $\mathrm{O}$ não atendimento destes pré-requisitos geram dificuldades no uso do método, por isto a importância em atendê-los.

Em relação às dificuldades metodológicas do uso do QFD, não relacionadas aos pré-requisitos, foi identificada a quantidade de 114 . Em mais de $78 \%$ dos casos estas dificuldades estão relacionadas com a aplicação da matriz da qualidade. Deste modo, verifica-se que o modelo proposto deve auxiliar na elaboração da matriz da qualidade.

Foram identificadas 196 citações sobre recomendações para reduzir dificuldades na utilização do QFD. Em quase dois terços dos casos estas recomendações estão relacionadas a resolver as dificuldades na elaboração das etapas da matriz da qualidade (mais de $64 \%$ dos casos) como por exemplo recomendações para ajudar na definição das correlações entre as QEs (qualidades exigidas) e CQs (características da qualidade) em quase $15 \%$ dos casos.

\section{Resultados da pesquisa de campo (coleta de dados atual]}

Das 21 empresas usuárias do QFD, apenas sete delas responderam questionário e quatro empresas não aceitaram participar do estudo, por terem interrompido o uso do método. As informações destas empresas seriam importantes para verificar quais as razões e dificuldades que as fizeram desistir do método, mas não foi possível investigar tal fato. As empresas participantes eram dos seguintes setores: embalagens, produtos alimentícios, automobilístico, eletrodomésticos, produtos químicos, serviços: vendas, manutenção e modernização de elevadores, metalúrgica básica; a grande maioria localizada no estado de São Paulo e uma em Minas Gerais. Deve-se observar que, embora tenha sido mencionada no texto a quantidade de empresas, o objetivo disto não é verificar a freqüência de citação (mesmo porque a amostra de usuários é pequena), mas sim a natureza das colocações feitas por elas na utilização do QFD. 
sobre os recursos necessários para introduzir o QFD, três empresas responderam esta questão; os resultados destas respostas indicam que as empresas não tinham uma previsão dos recursos necessários para a introdução.

As expectativas no uso do QFD foram confirmadas para as dificuldades de introdução (três empresas) e no uso cotidiano do QFD (três empresas). O método também gerou parte dos benefícios esperados pelas sete empresas de modo que, de forma geral, os resultados têm sido satisfatórios para as empresas que responderam aos questionários.

\section{Resultados da revisão do levantamento de campo realizada no passado}

Foram realizadas no passado quatro entrevistas em empresas consideradas como referência no uso do QFD no Brasil (CARNEVALLI; MIGUEL, 2003). As empresas pertenciam aos seguintes setores: alimentos (foram entrevistadas o "Consultor de Desenvolvimento de Novos Produtos" e o "Gerente de Desenvolvimento de Embalagem"); embalagens (sendo entrevistados a "Consultora de Desenvolvimento de Produtos", o "Analista de Processo de Fabricação" e o "Analista de Desenvolvimento de Produtos"); automotivo (foram entrevistados o "Gerente de Qualidade", o "Engenheiro da Qualidade" e o "Analista da Qualidade Desenvolvimento de Novos Projetos"); máquinas e equipamentos (foram entrevistados o "Consultor de Normas, Regulamentos e Engenharia de Materiais" e o "Gerente de Projeto e Desenvolvimento de Produto"). pela empresa, pois tal conhecimento está concentrado num departamento ou em poucos funcionários;

- Fazer com que o QFD contribua para garantir a qualidade do produto e com que contribua para diminuir o risco do projeto, a partir do momento em que a empresa identifica quais requisitos dos clientes e características da qualidade são prioritárias para atendê-los.

Também foi identificada uma dificuldade, que vale tanto para a alta direção quanto para os funcionários: um impacto "negativo" ao visualizarem uma matriz do QFD pela primeira vez. Segundo o entrevistado, "as pessoas se assustam com a matriz e com os símbolos das correlações, por não saberem os seus significados, prejulgando o método como complicado, antes de receberem as devidas orientações".

\section{Aplicação do Projeto Axiomático}

A seguir são apresentadas as etapas na utilização do AD para desenvolvimento do modelo proposto.

\section{Definição dos Clientes do QFD e escolha dos CAs a serem atendidos}

Para se iniciar a aplicação do projeto axiomático, deve-se primeiro definir o domínio do cliente, com a definição dos seus clientes e dos seus atributos em relação ao QFD. Estes são aqueles que são afetados pela aplicação do método, de forma direta ou indireta, sendo os clientes diretos os usuários (membros da equipe que aplicam o método), os gerentes das áreas funcionais envolvidos com o QFD, mas que não fazem parte da equipe (eles também são influenciados pelo método por haver a possibilidade de terem seus subordinados dentro da equipe) e os membros da alta administração da empresa, que devem fornecer recursos e apoio para o método e espera resultados

Foram identificadas, nestas entrevistas, as seguintes expectativas dos usuários do QFD quando iniciaram a aplicação do método: "auxiliar no processo de desenvolvimento de produtos"; "identificar e interpretar as necessidades dos clientes e traduzi-las em especificações de projetos"; "tornar-se líder no desenvolvimento de produtos", "desenvolver um produto que atenda vários dos seus mercados pelo mundo".

Para o presente trabalho, uma nova análise mais aprofundada das transcrições destas entrevistas foi feita, indicando outras expectativas dos usuários do QFD, não apresentadas em Carnevalli e Miguel (2003). Entre elas estão:

- Entender as correlações entre características de qualidade e processos produtivos e os seus efeitos no produto final;

- Difundir o conhecimento de desenvolvimento de produto satisfatórios com a sua aplicação. Os clientes indiretos são os indivíduos que adquirem produtos e serviços resultantes dos projetos desenvolvidos com o QFD (consumidores dos produtos da empresa). A partir da definição de quais são os clientes do QFD tem-se assim os seus CAs definidos com a análise da literatura, pesquisa de campo e revisão de entrevistas realizadas no passado pelos autores (apresentados resumidamente nos tópicos 3.1 a 3.3):

- Os atributos dos usuários definidos foram: $\mathrm{CA}_{1 \text { usuário: }}$ : "Pouca dificuldade para introduzir o método"; $\mathrm{CA}_{2 \text { usuário }}$ : "Ser o QFD um método eficaz para ajudar no processo de


para documentar e difundir o conhecimento do processo de desenvolvimento de produto"; $\mathrm{CA}_{4 \text { usuário }}$ "Pouca dificuldade para o uso do QFD no cotidiano da empresa".

- Os gerentes das áreas envolvidos com o QFD tem os 
seguintes atributos: $\mathrm{CA}_{1 \text { gerentes }}$ : "Ser o QFD um método eficaz para ajudar no planejamento da concepção do produto" e CA 2 gerentes: “A utilização do QFD deve gerar resultados melhores que os gerados pelo modo atual de trabalho no departamento" (em relação à priorização das especificações).

- Os atributos da alta administração são: CA 1 administracão: "Ter resultados de melhorias operacionais com o QFD" e $\mathrm{CA}_{2 \text { administração }}$ "O QFD gere o retorno do investimento de modo que justifique os seus custos de aplicação (custo de prevenção)".

- Os clientes da empresa tem os seguintes atributos: $\mathrm{CA}_{1 \text { clientes }}$ : "Receber produto que atenda as suas necessidades".

Após identificar os atributos dos clientes deve-se buscar atender ao maior número possível de atributos por meio da escolha do FR1. Para isto analisou-se a possibilidade de atender cada CA. Na impossibilidade de atender a todos, buscou-se o CA de maior relevância, o qual também possibilitaria atender totalmente ou parcialmente os demais. Assim, resolveu-se atender o atributo $\mathrm{CA}_{4 \text { usuário, pois ele }}$ também atende parcialmente aos atributos $\mathrm{CA}_{1 \text { usuário }}, \mathrm{CA}_{2}$ usuário, $\mathrm{CA}_{1 \text { gerentes, }} \mathrm{CA}_{2 \text { gerentes }}, \mathrm{CA}_{1 \text { administração }}, \mathrm{CA}_{1 \text { clientes }}$ a partir do momento que cria meios para avaliar alguns dos resultados da aplicação do QFD e resolve aspectos metodológicos de aplicação do método.

\section{Definição do FR1 e do DP1}

Identificado o CA a ser satisfeito, deve-se iniciar pela definição do FR1. Deste modo, o FR1 para atender o $\mathrm{CA}_{4 \text { usuário }}$ "Pouca dificuldade para o uso do QFD no cotidiano da empresa" deve estar relacionado com a redução das dificuldades metodológicas e de trabalho em grupo. Assim, o primeiro requisito funcional deve ser FR1: "Minimizar dificuldade de utilização do QFD”. Na elaboração do modelo de aplicação do QFD não estão sendo consideradas as premissas "externas" ao método, tais como os recursos necessários para aplicar o método, pois estas premissas também são encontradas na introdução de outros métodos complexos.

Após a definição do FR1, deve-se buscar no domínio físico um DP que o atenda. Podem existir várias alternativas para se atender o FR1 e, entre elas, foi desenvolvido o DP1: "Modelo de aplicação que minimize dificuldade do uso do QFD".

\section{Definição do $2^{\circ}$ nível da estrutura hierárquica}

Com a definição do primeiro nível da estrutura hierárquica, os FR1 e DP1 devem ser desdobrados em níveis mais detalhados, de forma hierarquizada até que os DPs estejam suficientemente detalhados para serem implementados.
Assim, a partir da definição do DP1 retorna-se para o FR1 para ser detalhado. Para isto deve-se considerar a seguinte questão: quais dificuldades de utilização do QFD devem ser minimizadas pelo modelo de aplicação? A identificação destas dificuldades foi feita por meio de análise da literatura. Nesta revisão, verificou-se que as dificuldades do uso do QFD estão relacionadas às etapas de elaboração da matriz da qualidade; dificuldades com o trabalho em grupo; elaborar o modelo conceitual e definir meios de avaliar o desenvolvimento e resultados da aplicação do QFD. No caso do trabalho em grupo, antes de resolver as dificuldades, é necessário verificar os reais motivos que levam à sua utilização no QFD, para verificar se existem outras possibilidades de DPs. Para isto, deve-se considerar dois aspectos:

- Primeiro, segundo Ohfuji et al. (1997), uma das necessidades que levou ao desenvolvimento do QFD era resolver falhas no fluxo de informações entre os departamentos do projeto, pois ocorria perda de informações no desenvolvimento seqüencial de um novo produto.

- Segundo, a orientação metodológica do QFD, relacionada à perspectiva pluralista, busca as diferentes visões e enfoques dos departamentos envolvidos no desenvolvimento do produto (CHENG, 2003). Deste modo, verifica-se a busca da integração das informações e do conhecimento dos departamentos.

Com as considerações anteriores, FR1 foi detalhado nos seguintes requisitos: FR11: "Elaborar a matriz da qualidade"; FR12: "Integrar as áreas funcionais envolvidas com o projeto"; FR13: "Elaborar o modelo conceitual" e FR14: "Monitorar a aplicação do QFD".

Com a definição do segundo nível hierárquico de FR1 retorna-se ao DP1 para detalhá-lo de modo que atenda a estes requisitos, sendo assim detalhado nos seguintes parâmetros de projeto: DP11: “Avaliação de soluções propostas na literatura sobre a matriz"; DP12: "Utilização de equipes multifuncionais"; DP13: "Caracterização dos objetivos da aplicação" e DP14: "Avaliação dos resultados da aplicação".

Para definição do DP11 foi considerada a recomendação de Cheng (2003) de levar em conta a literatura como exemplo, mas não como regra na elaboração das tabelas e matrizes do QFD. Assim, ao invés de utilizar-se a literatura como regra, deve-se realizar a avaliação dos vários exemplos de soluções propostas nesta literatura para melhorar o QFD. Deste modo, possibilita identificar as melhores soluções para resolver as dificuldades na elaboração da matriz da qualidade, mostrando-se eficaz para atender o FR11.

Para definir o DP12 não foi identificada, na literatura sobre o QFD, uma solução diferente de se trabalhar em equipe. O parâmetro foi definido como DP12: "Utilização de equipe multifuncional", sendo este considerado pré- 
requisito no trabalho de Herrmann et al. (2006). Entretanto, é importante lembrar que nada impede as empresas de aplicarem o QFD com uma equipe formada por membros de um único departamento, mas tal aplicação não é desejável, neste caso, pois não atende o FR12. O DP12 também atende FR13, pois o uso de equipes multifuncionais soma o conhecimento necessário para a redução da dificuldade de se fazer o modelo conceitual, que está relacionado aos objetivos do projeto, tipo de produto e processo de fabricação.

\section{Definição do $3^{\text {o }}$ nível da estrutura hierárquica}

Com a definição do $2^{\circ}$ nível na estrutura hierárquica, verifica-se o desdobramento de FR1 em quatro diferentes ramos: FR11, FR12, FR13 e FR14. Cada um destes ramos vai contribuir para detalhar de forma específica o DP1. Por esta razão, o detalhamento do parâmetro de projeto para o 3o nível é realizado de forma separada, por ramo, visando maior objetividade. Por questões de extensão do trabalho, é apresentado resumidamente o detalhamento dos ramos FR11, FR12, FR13 e FR14.

Para detalhar o FR11, "Elaborar a matriz da qualidade", devese considerar o DP11: “Avaliação de soluções propostas na literatura sobre a matriz" por meio da seguinte questão: quais são as dificuldades de elaborar a matriz da qualidade que serão minimizadas pelas soluções propostas na literatura? A resposta a esta questão

Segundo Cheng (2003), o modelo conceitual depende dos objetivos do projeto, características da indústria, do produto e do processo de fabricação. Analisando estes fatores, deve-se definir o modelo conceitual proposto considerando inicialmente a sua função principal "atingir os objetivos do projeto"; assim o DP13 será: "Caracterização dos objetivos da aplicação". O DP13 também atende o FR14, pois a caracterização dos objetivos do uso do QFD, que ajuda a definir os resultados que devem ser monitorados na aplicação.

A definição do DP14: “Avaliação dos resultados da aplicação" foi feita considerando a recomendação de Lowe e Ridgway (2000), em termos da necessidade de verificar os resultados do QFD, pois um dos modos de monitorar as etapas da aplicação é por meio da análise dos resultados.

Com a definição dos FRs e DPs do 2o nível realiza-se a matriz de projeto para verificar se o relacionamento entre os FRs e DPs não viola o axioma 1. Essa matriz é dada por:

$$
\left\{\begin{array}{l}
F R 11 \\
F R 12 \\
F R 13 \\
F R 14
\end{array}\right\}=\left[\begin{array}{cccc}
X & 0 & 0 & 0 \\
0 & X & 0 & 0 \\
0 & X & X & 0 \\
0 & 0 & X & X
\end{array}\right] \times\left\{\begin{array}{l}
D P 11 \\
D P 12 \\
D P 13 \\
D P 14
\end{array}\right\}
$$

Segundo El-Haik (2005) na matriz de projeto, " $x$ " indica forte relacionamento entre FR e DP. Assim, verifica-se que esta é uma matriz de projeto decoupled, satisfazendo, portanto, o axioma 1. é dada por meio da análise da literatura consultada, em que se verificou que as principais dificuldades para elaborar a matriz da qualidade estão relacionadas com a definição da qualidade projetada (RAMASAMY; SELLADURAI, 2004), realização das correlações (KARSAK, 2004), interpretação dos requisitos dos clientes (CHAN; WU, 2005); tradução da QE em CQ (FUNG et al., 2006); identificação dos requisitos mais importantes (YAN et al., 2005) e trabalhar com matrizes grandes (DIKMEN et al., 2005).

Após esta análise foi possível decompor o FR11 considerando os seguintes itens: FR111: "Definir a qualidade exigida do projeto"; FR112: "Desdobrar os requisitos dos clientes em características da qualidade"; FR113: "Definir os limites da matriz da qualidade"; e FR114: "Definir a qualidade projetada".

Com a definição dos FR111, FR112, FR113 e FR114 retorna-se para o DP11 e realiza-se o seu detalhamento para atender a estes requisitos funcionais definindo os seguintes parâmetros de projetos: DP111: "Pesquisa de mercado focada para o uso do QFD”; DP112: “Organização do conhecimento técnico para desdobrar a QE em CQ"; DP113: "Priorização das QEs que farão parte da matriz"; e DP114: "Priorização das CQs para a qualidade projetada".

O FR111 envolve desde a realização de pesquisa de mercado até a definição da qualidade planejada. Por esta razão, o DP111 foi definido considerando que para definir a qualidade exigida do projeto é necessário inicialmente identificar os requisitos dos clientes coletando dados destes clientes. Além disso, uma empresa que realiza pesquisa de mercado focada para o uso do QFD apresenta melhores resultados, como a redução do lead-time de projeto (CRISTIANO et 
al., 2001). O DP111 também satisfaz o FR112 e o FR114, pois a pesquisa de mercado pode envolver o processo de benchmarking, o que influencia na definição das CQs e qualidade projetada.

Com a análise da literatura verificou-se que para satisfazer o FR112 existem várias soluções que podem ser aplicadas, mas não foi identificada uma solução ideal para a definição das CQs. Uma solução eficiente seria a combinação de algumas das recomendações de Fehlmann (2003) (uso do diagrama de Ishikawa); Rahim e Baksh (2003) (análise de todos os dados disponíveis dentro da empresa); e Cheng e Melo Filho (2007) (realização de brainstorming). Deste modo, para definir o DP112 considerou-se uma solução ampla, a qual será detalhada no nível subseqüente. Para isto, verificou-se que as recomendações dos autores se referem a organização dos conhecimentos científicos e empíricos da empresa para definir as CQs. O DP112 também atende ao FR114, pois a organização do conhecimento técnico influencia na definição da qualidade projetada.

O FR113 surge da necessidade de se definir o tamanho da matriz da qualidade, que não deve ser muito grande para não gerar dificuldades de trabalho, nem muito pequena para não prejudicar a eficácia do projeto. Para atender ao FR113, considerou-se a recomendação de Marsot (2005), pela qual a equipe deve fazer uma priorização das QEs com base na sua experiência.

Para atender ao FR114 deve-se considerar quais são as etapas necessárias para definir a qualidade projetada, que envolve a análise das correlações CQs versus CQs; QEs versus CQs e a realização do benchmarking técnico. Esta análise tem dois objetivos: identificar as CQs prioritárias e os seus valores nominais para atingir os objetivos do projeto. Seguindo esta lógica, primeiro deve-se definir as CQs prioritárias para posteriormente definir os seus valores nominais.

Com a definição dos FRs e DPs do $3^{-}$nível do ramo FR11, realiza-se a matriz de projeto para verificar se o relacionamento entre os FRs e DPs não viola o axioma 1, obtendo assim:

$$
\left\{\begin{array}{l}
F R 111 \\
F R 112 \\
F R 113 \\
F R 114
\end{array}\right\}=\left[\begin{array}{cccc}
X & 0 & 0 & 0 \\
X & X & 0 & 0 \\
0 & 0 & X & 0 \\
X & X & 0 & X
\end{array}\right] \times\left\{\begin{array}{l}
D P 111 \\
D P 112 \\
D P 113 \\
D P 114
\end{array}\right\}
$$

Verifica-se então que a matriz de projeto é decoupled, satisfazendo o axioma 1.

Como o FR12: "Integrar as áreas funcionais envolvidas com o projeto", foi atendido pelo DP12: "Utilização de equipes multifuncionais", deve-se verificar quais dificuldades de se trabalhar com equipes podem ser minimizadas pelo modelo de aplicação, visando auxiliar a integração. Pela literatura, verifica-se que o trabalho em equipe gera várias dificuldades, tais como: conflitos na equipe (MIGUEL, 2003), falta de consenso (LOWE; RIDGWAY, 2000), obtenção de comprometimento da equipe (MARTINS; ASPINWALL, 2001) e lidar com equipes muito grandes (GINN; ZAIRI, 2005). Com estas considerações, o FR12 pode ser detalhado em: FR121: "Resolver conflitos na equipe"; FR122: "Atingir o consenso na equipe"; FR123: "Definir o tamanho da equipe" e FR124: "Conseguir o comprometimento".

Para se detalhar o DP12, de modo que satisfaça os FR121, FR122, FR123 e FR124, considerou-se, além dos dados da literatura específica sobre QFD, a análise de trabalhos sobre gestão de conflitos e dados da pesquisa de campo atual. O DP12 foi detalhado, estabelecendo os seguintes itens: DP121: "Realização de ações de gestão de conflitos"; DP122: "Consenso atingido pelo líder da equipe"; DP123: "Participação apenas das pessoas necessárias em cada etapa"; e DP124: "Definição clara das responsabilidades dos membros da equipe".

Para se definir o DP121 foi considerado que a gestão adequada dos conflitos dentro da equipe melhora o seu desempenho (DIONNE et al., 2004). O DP121 também satisfaz o FR122, pois a gestão de conflitos influencia no atingimento de consenso na equipe.

Para se definir o DP122 considerou-se a definição feita por Guimarães (2003) sobre as funções do líder do projeto, entre elas a de buscar o consenso dentro da equipe.

Para se definir o tamanho da equipe (FR123) de modo a evitar equipes grandes, deve-se considerar os seguintes aspectos: não existe um número fixo de participantes na equipe do QFD, pois segundo Cheng et al. (1995) e Carnevalli e Miguel (2003) o número de participantes varia conforme a etapa do QFD. O aspecto mais importante é que em cada etapa estejam presentes na equipe as pessoas que têm o conhecimento necessário para realizá-las.

Para definir o DP124, considerou-se a proposta de Herrmann et al. (2006), de que é necessário definir claramente as responsabilidades da equipe, pois é a partir do momento que cada membro do grupo tem suas responsabilidades definidas que se pode cobrar e avaliar o seu comprometimento, para o cumprimento destas responsabilidades.

Com a definição dos FRs e DPs do 3ํ nível do ramo FR12, realiza-se a matriz de projeto para verificar se o relacionamento entre os FR121, FR122, FR123, FR124 com os DP121, DP122, DP123 e DP124 não viola o axioma 1, obtendo-se assim: 


$$
\left\{\begin{array}{l}
F R 121 \\
F R 122 \\
F R 123 \\
F R 124
\end{array}\right\}=\left[\begin{array}{cccc}
X & 0 & 0 & 0 \\
X & X & 0 & 0 \\
0 & 0 & X & 0 \\
0 & 0 & 0 & X
\end{array}\right] \times\left\{\begin{array}{l}
D P 121 \\
D P 122 \\
D P 123 \\
D P 124
\end{array}\right\}
$$

Como pode ser visto, verifica-se que a matriz de projeto é decoupled, satisfazendo assim o axioma 1.

O FR13: "Elaborar o modelo conceitual", foi atendido pelo DP13: "Caracterização dos objetivos da aplicação", surgindo a seguinte questão: como elaborar o modelo conceitual de modo que atenda aos objetivos do projeto? Para responder a esta questão deve-se avaliar dois fatores: primeiro o argumento de Cheng (2003), de que o modelo conceitual (do QFD) deve ser formado pelas matrizes necessárias para atender aos requisitos dos clientes, ou seja, determinar qual é o número de matrizes necessárias e quais são elas; segundo, a necessidade de se definir a distribuição das matrizes do QFD de forma adequada no modelo conceitual, para aplicar o método na seqüência certa, visando atingir os objetivos do projeto.

desenvolvimento é um derivativo, plataforma ou serviço ele necessita de diferentes números ou tipo de matrizes.

Para satisfazer o FR132 o fator mais importante a ser considerado é a análise do processo e fabricação, a qual permite verificar toda a seqüência de obtenção do produto.

Com a definição dos requisitos funcionais e parâmetro de projeto do ramo FR13 realiza-se a matriz de projeto para verificar se ela não viola o axioma1, obtendo-se assim:

$$
\left\{\begin{array}{l}
F R 131 \\
F R 132
\end{array}\right\}=\left[\begin{array}{ll}
X & 0 \\
0 & X
\end{array}\right] \times\left\{\begin{array}{l}
D P 131 \\
D P 132
\end{array}\right\}
$$

Na matriz de projeto só foram considerados os relacionamento fortes entre FRs e DPs, gerando uma matriz de projeto uncoupled, que satisfaz o axioma 1.

Sendo o FR14, "Monitorar a aplicação do QFD” atendido pelo DP14, "Avaliação dos resultados da aplicação", a questão que surge é quais resultados do QFD devem ser monitorados para verificar se o método está sendo aplicado adequadamente e se o resultado da aplicação justifica a sua continuidade. Deste modo, existem dois grupos de resultados do QFD a serem analisados. O primeiro grupo se refere à análise da execução da aplicação e o segundo grupo está relacionado aos benefícios gerados após a aplicação, pois na pesquisa de campo atual verificou-se que a alta administração espera "Ter resultados de melhorias operacionais com o QFD” (CA 1 administração $)$.

Analisando-se estas duas conside-

Após a análise desses dois fatores pode-se detalhar o requisito funcional FR13 em: FR131: "Definir o número de matrizes necessárias para atingir os objetivos"; e FR132: "Definir a disposição das matrizes".

Para atender ao FR131 e ao FR132 o DP13 foi detalhado nos seguintes itens do $3^{\circ}$ nível da estrutura hierarquizada: DP131, "Definição do número de matrizes pelo tipo de produto"; e DP132, "Caracterização da sua seqüência de obtenção do produto" (CHENG, 2003).

Para definir os parâmetros de projeto DP131 considerou-se novamente o trabalho de Cheng (2003), segundo o qual o modelo conceitual depende dos objetivos do projeto (já atendido no parâmetro DP13), características da indústria, do produto em desenvolvimento e do processo de fabricação, e tipo de modelo (principal ou auxiliar).

Para satisfazer o FR131 o fator importante é o tipo de modelo conceitual, ao qual está relacionado às características do produto em desenvolvimento. Se o produto em rações, o FR14 foi detalhado levando-se em conta os seguintes itens: FR141: "Monitorar os resultados durante a aplicação do QFD”; FR142: "Monitorar os resultados após a aplicação do QFD”.

Para atender aos requisitos do ramo FR14, o DP14 foi detalhado considerando os seguintes itens: DP141: "Avaliação dos resultados das etapas de aplicação" (SANFORD, 2005; LOWE; RIDGWAY; 2000) e DP142: "Avaliação dos benefícios tangíveis do projeto".

Para atender ao requisito funcional FR142 deve-se avaliar quais são os possíveis resultados que se pode monitorar sobre o uso do método. Para a resposta a esta questão, devem-se considerar os seguintes aspectos:

- A partir da análise da literatura verificou-se que os benefícios do QFD podem ser classificados em quatro grupos: benefícios tangíveis ou intangíveis referentes à melhoria do projeto, ou benefícios tangíveis ou intangíveis fora do projeto. Com base nesta análise e 
para justificar o uso cotidiano do QFD é importante monitorar resultados que possam ser quantificados, devendo assim considerar os benefícios tangíveis nesta análise.

- Na pesquisa de campo atual verificou-se que a alta administração esperava ter retorno do investimento com a aplicação do QFD e que o método gerasse resultados de melhorias operacionais. Para atender a este aspecto, mesmo que parcialmente, devem-se monitorar os benefícios tangíveis referentes à melhoria do projeto.

- O sucesso de um projeto também é influenciado por aspectos externos, tais como o crescimento econômico do mercado, e a estratégia dos concorrentes, não sendo possível verificar uma vantagem competitiva gerada pelo QFD, como verificou Damante (1997). Deste modo, os benefícios relacionados ao aumento das vendas e da participação no mercado não devem ser avaliados.

- Segundo o argumento de Cheng (2003), os resultados de um projeto de desenvolvimento de produto são conseqüência do uso em conjunto de várias técnicas e métodos, considerando impraticável a separação e medição somente dos resultados do QFD. Assim, como não é possível separar confiavelmente os resultados exclusivos do QFD no projeto, deve-se medir o quanto o projeto melhorou após a introdução do QFD de modo a comprovar, indiretamente, mas de forma mensurável a viabilidade do seu uso. Deste modo, o parâmetro de projeto foi definido como DP142: "Avaliação dos benefícios tangíveis no projeto".

Com a definição dos requisitos e parâmetros do ramo do 3 o nível da estrutura hierárquica, realiza-se a matriz de projeto para verificar se não viola o axioma 1 , obtendo-se a matriz:

$$
\left\{\begin{array}{l}
F R 141 \\
F R 142
\end{array}\right\}=\left[\begin{array}{ll}
X & 0 \\
0 & X
\end{array}\right] \times\left\{\begin{array}{l}
D P 141 \\
D P 142
\end{array}\right\}
$$

Mais uma vez, verifica-se que é uma matriz uncoupled, o que satisfaz o axioma 1.

Finalmente, cabe destacar que neste trabalho o desenvolvimento dos três primeiros níveis da estrutura hierarquizada do modelo proposto indica a viabilidade do uso do projeto axiomático para o desenvolvimento de um modelo de aplicação do QFD. Outros desdobramentos dos FRs e DPs, e a elaboração da matriz de projeto consolidada, não foram apresentados neste trabalho, e devem ser discutidos em um trabalho futuro. O modelo completo pode envolver cinco níveis da estrutura hierarquizada, totalizando 44 FRs e 44 DPs analisados em 14 matrizes de projeto. As Figuras 4 a 6 apresentam resumidamente a estrutura de 5 níveis hierarquizados desenvolvida e o diagrama de módulo e junção. Con- cluído o modelo conceitual proposto, utilizou-se a matriz de projeto consolidada para verificar a sua validade, o que pode ser confirmado. Para definir a seqüência de aplicação do modelo foi utilizado o diagrama de módulos e junção, ilustrado na Figura 6.

Verifica-se na Figura 6 que o bloco M11 apresenta uma relação de soma (aplicação em paralelo) com o bloco M14, o qual apresenta relação de controle (aplicação em seqüência) com o bloco M13 e este com o bloco M12. Isto indica que as soluções do bloco M11 (para a matriz da qualidade) podem ser aplicadas em paralelo com as soluções dos blocos M12 (relacionadas a equipe), M13 (relacionadas ao modelo conceitual) e M14 (para monitorar a aplicação do QFD), nesta ordem.

\section{CONCLUSÕES}

Primeiramente pode ser concluído que a revisão da literatura representou dados e informações relevantes na elaboração do modelo conceitual proposto. A utilização do diagrama de afinidades e o diagrama em árvore permitiram organizar e agrupar de forma hierárquica as informações sobre os benefícios, as dificuldades, recomendações e prérequisitos do uso do QFD, extraídos da literatura, facilitando posteriormente a utilização destes dados na aplicação do AD.

Os resultados do levantamento de campo e da reanálise dos dados de entrevistas completaram os dados da revisão bibliográfica com informações empíricas de indivíduos experientes na aplicação do QFD e que tiveram diferentes contatos com o método (líder da equipe do QFD, membro da equipe, etc.) indicando assim diferentes expectativas das empresas usuárias. Estes dados foram utilizados para aplicar o método do AD no desenvolvimento do modelo proposto. No levantamento de campo também se verificou que reduzir as dificuldades do uso cotidiano do QFD faz parte dos atributos dos clientes, justificando assim a aplicação do AD com este objetivo. Com esta análise, conclui-se que os nove atributos dos clientes identificados para o QFD estão relacionados aos resultados da aplicação e à redução das dificuldades de introdução e utilização do método (domínio do cliente). Na impossibilidade de atender a todos, buscouse o CA mais importante $\left(\mathrm{CA}_{4 \text { usuário }}\right)$, o qual também permite atender total ou parcialmente aos demais.

Definido o domínio do cliente, estruturou-se o domínio funcional e o domínio físico onde os FRs são relativos às dificuldades do uso do QFD, identificadas na literatura a serem reduzidas, pelas soluções que são os DPs, também identificadas na sua maioria na mesma literatura estudada, para atender o $\mathrm{CA}_{4}$ usuário . Conclui-se que as principais dificuldades a serem resolvidas estão relacionadas à matriz da qualidade, a elaborar modelo conceitual, ao trabalho em 
Figura 4: Representação gráfica da estrutura hierárquica de FRs.



Figura 5: Representação gráfica da estrutura hierárquica de DPs.

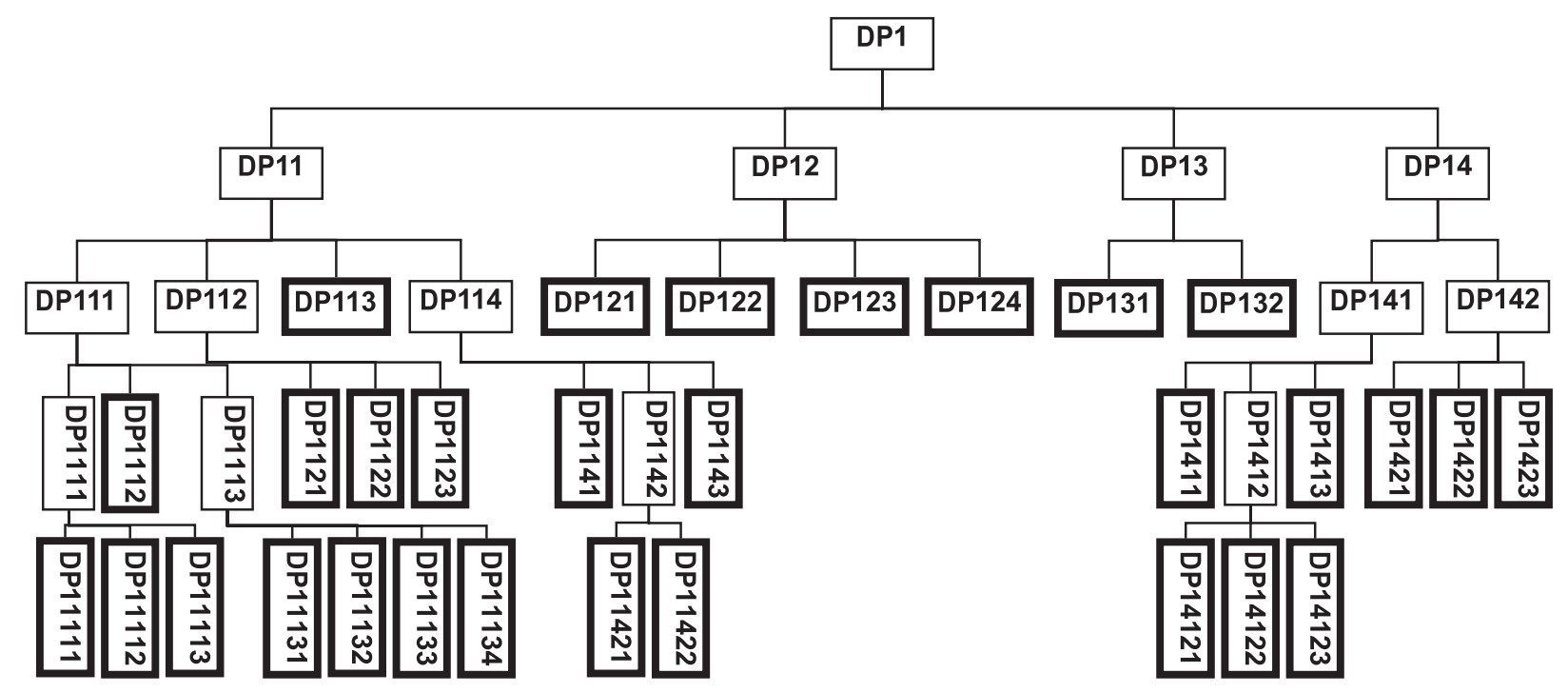


equipe e a avaliação da aplicação do QFD. Deste modo, o detalhamento dos requisitos funcionais e parâmetros de projetos até o 5o nível da estrutura hierarquizada no $\mathrm{AD}$ possibilitou a estruturação do modelo conceitual desenvolvido, confirmando a viabilidade do seu uso para cumprir o objetivo proposto.

Nessa fase do desenvolvimento do trabalho também é possível concluir que a utilização da matriz de projeto consolidada para verificar a validade do modelo desenvolvido e o diagrama de módulos e junção para definir a seqüência de aplicação do modelo foram suficientemente estabelecidos.

Cabe destacar ainda que, em um nível conclusivo que necessita de validação mais extensiva, existem pontos concordantes (dificuldades e expectativas no uso do QFD, benefícios, etc.) levantados tanto na literatura quanto no trabalho de campo. A maior parte das limitações no uso do QFD estão associadas ao seu modus operandi, sendo que um maior esforço, em termos de pesquisa, deve ser empreendido nessa direção. Ainda com algumas limitações, o modelo conceitual desenvolvido apresenta uma proposta de monitoramento da aplicação e avaliação parcial dos resultados tangíveis do projeto indiretamente causados pelas ações que o uso do QFD geraram. Necessita, no entanto, de uma aplicação visando sua validação empírica.

Embora necessite de um teste de validade empírica, o modelo conceitual proposto pode ser considerado para aplicações do QFD de melhoria ou reprojeto de produtos, processos e serviços.

Figura 6: Diagrama de módulo e junção do modelo proposto.






\section{Artigo recebido em 20/06/2007 \\ Aprovado para publicação em 02/01/2008}

\section{- Referências}

CARNEVALLI, J. A.; MIGUEL, P. A. C. Empresas de Referência na Utilização do Desdobramento da Função Qualidade. In: CONGRESSO BRASILEIRO GESTÃO DE DESENVOLVIMENTO DE PRODUTO, 4., 2003, Gramado, Anais ... 2003. 1 CD-ROM.

CARNEVALLI, J. A. et al. Aplicação do QFD no desenvolvimento de produtos: levantamento sobre seu uso e perspectivas para pesquisas futuras. Gestão \& Produção, v. 11, n. 1, p. 33-49, 2004.

CHAN; L. K.; WU, M. L. A systematic approach to quality function deployment with a full illustrative example. Omega - The International Journal of Management Science, v. 33, n. 2, p. 119-139, 2005.

CHENG, L.C. et al. QFD: planejamento da qualidade. Belo Horizonte: Fundação Christiano Ottoni, 1995.

CHENG, L. C. QFD in product development: methodological characteristics and a guide for intervention. International Journal of Quality \& Reliability Management, v. 20, n. 1, p. 107-122, 2003.

CHENG, L.C.; MELO FILHO, L.D.R. QFD: Desdobramento da Função Qualidade na gestão de desenvolvimento de produtos. São Paulo: Editora Blucher, 2007.

COOPER, D.R.; SCHINDLER, P.S. Métodos de pesquisa em administração. Porto Alegre: Bookman, 2003.

CRISTIANO, J. J. et al. Key factors in the successful application of quality function deployment (QFD). IEEE Transactions on Engineering Management, v. 48 , n. 1 , p. $81-95,2001$

DAMANTE, F.C. Desdobramento da Função Qualidade: um estudo de sua aplicação no Brasil, 162 p. Dissertação (Mestrado em Administração) Faculdade de Economia, Administração e Contabilidade, USP, São Paulo, 1997.
DEVADASAN. D. et al. Theory and practice of total quality function deployment: A perpective from traditional pump-manufacturing environment. The TQM Magazine, v. 18, n. 2, p. 143-161, 2006

DIKMEN, I. et al. Strategic use of quality function deployment (QFD) in the construction industry. Building and Environment, v. 40 , n. 2, p. 245-255, 2005.

DIONNE, S. D., et al. Transformational leadership and team performance. Journal of Organizational Change Management, v. 17, n. 2, p. 177-193, 2004

EL-HAIK, B. S. Axiomatic Quality: Integrating Axiomatic Design With Six-Sigma, Reliability, And Quality Engineering. Hoboken, New Jersey: John Wiley \& Sons, Inc., 2005.

FEHLMANN; T. M. Strategic management by business metrics: An application of combinatory metrics. International Journal of Quality \& Reliability Management, v. 20, n. 1, p. 134-145, 2003.

FUNG, R. Y. K. et al. Estimating the functional relationships for quality function deployment under uncertainties. Fuzzy Sets and Systems, v. 157, n. 1, p. 98-120, 2006.

GINN, D.; ZAIRI, M. Best practice OFD application: an internal/external benchmarking approach based on Ford Motors' experience. International Journa of Quality \& Reliability Management, v. 22 , n. 1 , p. $38-58,2005$

GUIMARÃES, I. M. QFD, analisando seus aspectos culturais organizacionais. Banas Qualidade, v. 12, n. 128, p. 56-66, 2003.

HERRMANN, A. et al. An empirical study of quality function deployment on company performance. International Journal of Quality \& Reliability Management, $\mathrm{v}$ 23, n. 4 , p. $345-366,2006$
KARSAK, E. E. Fuzzy multiple objective programming framework to prioritize design requirements in quality function deployment. Computers $\mathcal{E}$ Industrial Engineering, v. 47, n. 2-3, p. 149-163, 2004.

LAGER, T. The industrial usability of quality function deployment: a literature review and synthesis on a metalevel. R\&D Management, v. 35, n. 4, p. 409-426, 2005

LOWE, A.; RIDGWAY, K. UK user's guide to quality function deployment. Engineering Management Journal, v. 10, n. 3, p. 147-155, 2000.

MARCONI, M. D. A.; LAKATOS, E.M. Técnicas de pesquisa: planejamento e execução de pesquisas, amostragens e técnicas de pesquisas, elaboração, análise e interpretação de dados. São Paulo: Atlas, 2002.

MARSOT, J. QFD: a methodological tool for integration of ergonomics at the design stage. Applied Ergonomics, v. 36, n. 2 , p. 185-192, 2005.

MARTíN, L. A. G. El estado actual de la investigación empírica sobre economía de la empresa: análisis de las publicaciones españolas. Papeles de Economía Española, n. 78-79, p. 302-317, 1999.

MARTINS, A.; ASPINWALL, E. M. Quality function deployment: an empirical stu$\mathrm{dy}$ in the UK. Total Quality Management, v. 12 , n. 5, p. 575-588, 2001.

MIGUEL, P. A. C. The state-of-the-art of the Brazilian QFD applications at the top 500 companies. International Journal of Quality \& Reliability Management, v. 20, n. 1, p.74-89, 2003.

OHFUJI, T. et al. Métodos de desdobramento da qualidade (1). Belo Horizonte: Fundação Christiano Ottoni. 1997.
OLIVEIRA, S. L. Tratado de metodologia científica: projetos de pesquisas, TGI, TCC, monografias, dissertações e teses. São Paulo: Pioneira, 1997.

RAHIM, A. R. A.; BAKSH, M. S. N Application of quality function deployment (QFD) method for pultrusion machine design planning. Industrial Management \& Data Systems, v. 103, n. 6, p. 373-387, 2003.

RAMASAMY, N. R.; SELLADURAI, V. Fuzzy logic approach to prioritise enginnering characteristics in quality function deployment (FLQFD). International Journal of Quality \& Reliability Management, v. 21, n. 9, p. 1012-1023, 2004.

SANFORD, J. How useful is QFD? Quality Progress, v. 38, n. 1, p. 51-59, 2005.

SHER, S. S. The Application of Quality Function Deployment (QFD) in Product Development - The Case Study of Taiwan Hypermarket Building. The Journal of American Academy of Business, v. 8, n. 2, p. 292-295, 2006.

SUH, N. P. The principles of design. New York: Oxford University Press. 1990.

SUH, N. P. Axiomatic Design. New York: Oxford University Press. 2001.

VOSS, C. et al. Case Research: case research in operations management. International Journal of Operations $\mathcal{E}$ Production Management, v. 22, n. 2, p. 195-219, 2002.

YAN, W. et al. A QFD-enabled product conceptualisation approach via design knowledge hierarchy and RCE neural network. Knowledge-Based Systems, v. 18, n. 6, p. 279-293, 2005. 


\section{- Agradecimentos}

Os autores agradecem o apoio recebido pela CAPES (bolsa de doutorado) e as empresas que participaram desse estudo. Também agradecem os pareceres dos revisores que contribuíram significativamente para o aperfeiçoamento deste artigo.

- Sobre os autores

\section{José Antonio Carnevalli}

UNIMEP

Núcleo de Gestão da Qualidade \& Metrologia; Faculdade de Engenharia, Arquitetura e Urbanismo, UNIMEP

End.: Rod. SP306, Km 1 - CEP 13450-000 - Santa Bárbara d' Oeste - SP

Tel. (19) 3124-1797

E-mail: jcarnevalli@hotmail.com

\section{Paulo Augusto Cauchick Miguel}

EPUSP

Departamento de Engenharia de Produção, Escola Politécnica da USP

Tel. (11) 3091-5363 ramal 476

E-mail: paulo.miguel@poli.usp.br

\section{Felipe Araújo Calarge}

Diretoria de Ciências Exatas e Tecnológicas

Universidade Nove de Julho - UNINOVE

Av. Francisco Matarazzo, 612 - Agua Branca - São Paulo

Tel.: (11)3665-9300

E-mail: fcalarge@uninove.br 